\title{
Homotopy Groups of the Combinatorial Grassmannian
}

\author{
L. Anderson \\ Texas A\&M University, \\ College Station, \\ TX 77843-3368, USA \\ Laura.Anderson@math.tamu.edu
}

\begin{abstract}
We prove that the homotopy groups of the oriented matroid Grassmannian $\operatorname{MacP}(k, n)$ are stable as $n \rightarrow \infty$, that $\pi_{1}(\Delta \operatorname{MacP}(k, n)) \cong \pi_{1}\left(G\left(k, \mathbb{R}^{n}\right)\right)$, and that there is a surjection $\pi_{2}\left(G\left(k, \mathbb{R}^{n}\right)\right) \rightarrow \pi_{2}(\Delta \operatorname{MacP}(k, n))$.
\end{abstract}

The theory of oriented matroids gives rise to a combinatorial analogue to the Grassmannian $G\left(k, \mathbb{R}^{n}\right)$. By thinking of an oriented matroid as a "combinatorial vector space," one is led to define the combinatorial Grassmannian (or MacPhersonian $\operatorname{MacP}(k, n)$ ) as a partially ordered set, whose order complex is hoped to have topology similar to that of $G\left(k, \mathbb{R}^{n}\right)$. While there are some immediate and natural correspondences between these two topological spaces, the topology of the MacPhersonian is, on the whole, a mystery.

The topology of $\operatorname{MacP}(k, n)$ and the direct limit $\operatorname{MacP}(k, \infty)$ is of interest from several perspectives. From the topological perspective, the $\operatorname{MacPhersonian} \operatorname{MacP}(k, \infty)$ is the classifying space for the combinatorial vector bundles (or matroid bundles) defined in [6]. From the combinatorial side, perhaps the most interesting aspect of $\operatorname{MacP}(k, n)$ is the subcomplex generated by elements which do not correspond to real vector spacesthe nonrealizable oriented matroids. Any statement on the "tameness" of $\operatorname{MacP}(k, n)$ suggests limits on the exoticness of nonrealizable oriented matroids. This paper will present some progress toward taming the MacPhersonian.

\section{Introduction}

The combinatorial Grassmannian was first defined in [6], and was discussed in more detail in [8]. Below is a brief introduction and summary of previously known results. 
For background material on oriented matroids, see [3]. Briefly put, an oriented matroid is a combinatorial model for a finite arrangement of oriented hyperplanes in $\mathbb{R}^{k}$. The idea is as follows: a set $\mathcal{A}=\left\{H_{1}, \ldots, H_{n}\right\}$ of oriented hyperplanes through the origin partitions $\mathbb{R}^{k}$ into a finite set of cones. The orientations allow us to describe each cone $C$ by a sign vector $v_{C} \in\{+, 0,-\}^{n}$ : the $i$ th component of $v_{C}$ says whether $C$ is on the positive, negative, or 0 side of $H_{i}$. Let $\mathcal{V}_{\mathcal{A}}$ be the set of all such sign vectors arising from the arrangement $\mathcal{A}$. Certain combinatorial axioms are satisfied by $\mathcal{V}_{\mathcal{A}}$ for any oriented hyperplane arrangement $\mathcal{A}$. (The complete axioms are listed in [3, Section 3.7].) Any set $\mathcal{V}$ of sign vectors of length $n$ satisfying these axioms is called an oriented matroid with $n$ elements.

We should note that this model allows identical copies of hyperplanes (which simply means that for some $i, j \in\{1,2, \ldots, n\}$, each vector $v \in \mathcal{V}$ has $v_{i}=v_{j}$ ) and the "degenerate hyperplane" $0^{\perp}$ (i.e., an element $i$ of $[n]$ with $v_{i}=0$ for all $v \in \mathcal{V}$ ). ("Degenerate hyperplanes" are called loops in [3].) The set of "degenerate hyperplanes" is the zero set of the oriented matroid.

Not every oriented matroid is realizable as an arrangement of hyperplanes. However, Folkman and Lawrence showed (see [4] and [3, Section 1.4]) that all oriented matroids are "close" to being realizable. An arrangement of pseudospheres in $S^{k-1}$ is a collection $\left\{S_{1}, \ldots, S_{n}\right\}$ of subsets of $S^{k-1}$ such that each pair $\left(S^{k-1}, S_{i}\right)$ is homeomorphic to $\left(S^{k-1}, S^{k-2}\right)$ and the intersections of these subsets are lower-dimensional spheres satisfying the same combinatorial properties as an arrangement of diameters of $S^{k-1}$. (A special case is the set of diameters of the unit sphere given by an arrangement of hyperplanes in $\mathbb{R}^{k}$.) The Topological Representation Theorem of Folkman and Lawrence says that any oriented matroid can be realized by an arrangement of pseudospheres, and that every arrangement of pseudospheres gives an oriented matroid.

The axioms of oriented matroids are strong enough to describe much of the geometry of hyperplane arrangements. For instance, there is a natural notion of linear independence of elements of an oriented matroid, corresponding to independence of the normal vectors of a set of hyperplanes. The rank of an oriented matroid $M$ is defined to be the order of a maximal independent set of elements of $M$. We can also define an analogue to moving an arrangement of hyperplanes into more special position: this is called a specialization or weak map $M_{1} \leadsto M_{2}$ of oriented matroids (see [3, Section 7.7]). For instance, let $M_{1}$ be any oriented matroid, and let $M_{2}$ be the oriented matroid obtained from $M_{1}$ by setting some elements equal to 0 . Then $M_{2}$ is a specialization of $M_{1}$. This is a special case we will encounter often.

Let $\operatorname{MacP}(k, n)$ denote the set of all rank $k$ oriented matroids with elements $\{1,2, \ldots, n\}$. This set has a natural partial order, with $M_{1} \geq M_{2}$ if there is a weak map $M_{1} \leadsto M_{2}$. We associate a topological space to this set by taking the order complex $\Delta \operatorname{MacP}(k, n)$ of all chains in the partial order. Thus the vertices of $\Delta \operatorname{MacP}(k, n)$ are the rank $k$ oriented matroids with elements $\{1,2, \ldots, n\}$, and the $i$-simplices are all totally ordered $(i+1)$-tuples $\left\{M_{0} \leadsto M_{1} \leadsto \cdots \leadsto M_{i}\right\}$. Theorem 1.1 below will suggest how this space serves as a combinatorial analogue to the Grassmannian of $k$-planes in $\mathbb{R}^{n}$.

We also get a combinatorial analogue to the Grassmnnian of oriented $k$-planes in $\mathbb{R}^{n}$. We sketch this idea very briefly here: for a more complete background, see Section 3.5 in [3]. Consider once again an arrangement $\mathcal{A}$ of real oriented hyperplanes in $\mathbb{R}^{k}$. The orientation on each $H_{i}$ determines a canonical unit normal $v_{i}$ to $H_{i}$. An orientation on $\mathbb{R}^{k}$ 
gives an orientation for each ordered $k$-tuple of these unit normal vectors. In turn, we can specify an orientation of $\mathbb{R}^{k}$ and a combinatorial type of $n$-element hyperplane arrangement in $\mathbb{R}^{k}$ by giving an orientation on each ordered $k$-tuple in $\{1,2, \ldots, n\}$ in a way consistent with the Grassmann-Plücker relations. Such a map $\chi$ from ordered $k$-tuples in $\{1,2, \ldots, n\}$ to $\{+, 0,-\}$ is called a chirotope for the resulting arrangement. For any arrangement of hyperplanes in $\mathbb{R}^{k}$, the two orientations of $\mathbb{R}^{k}$ give two chirotopes $\chi$ and $-\chi$ for the arrangement. More generally, we can define chirotopes for oriented matroids. (See [3] for the combinatorial definition of chirotopes and the relation between chirotopes and oriented matroids.) A theorem of Las Vergnas [5] states that every oriented matroid has exactly two chirotopes $\chi$ and $-\chi$, and that the chirotope completely determines the oriented matroid. Thus we can define an oriented MacPhersonian $\widetilde{\operatorname{MacP}}(k, n)$, the poset of all rank $k$ chirotopes on $n$ elements, ordered by weak maps. The projection $\Delta \widetilde{\operatorname{MacP}}(k, n) \rightarrow \Delta \operatorname{MacP}(k, n)$ is easily seen to be a double cover.

There is a very natural map from elements in $G\left(k, \mathbb{R}^{n}\right)$ to the realizable oriented matroids in $\operatorname{MacP}(k, n)$. Let $V$ be an element of $G\left(k, \mathbb{R}^{n}\right)$, and let $\left\{H_{1}, \ldots, H_{n}\right\}$ be the coordinate hyperplanes in $\mathbb{R}^{n}$. Then $\mathcal{A}_{V}=\left\{V \cap H_{i}: i \in\{1,2, \ldots, n\}\right\}$ is an arrangement of $n$ (possibly degenerate) hyperplanes in $V \simeq \mathbb{R}^{k}$, and thus $\mathcal{A}_{V}$ gives an element of $\operatorname{MacP}(k, n)$. If $M$ is a realizable oriented matroid in $\operatorname{MacP}(k, n)$, let $U_{M}=\{V \in$ $G\left(k, \mathbb{R}^{n}\right): M$ is the oriented matroid given by $\mathcal{A}_{V}$. The set of all such $U_{M}$ gives a stratification of $G\left(k, \mathbb{R}^{n}\right)$.

Theorem 1.1 (from [6]). There is a triangulation of the real Grassmannian $G\left(k, \mathbb{R}^{n}\right)$ refining this stratification, giving a simplicial map

$$
c: G\left(k, \mathbb{R}^{n}\right) \rightarrow \Delta \operatorname{MacP}(k, n)
$$

which is canonical up to homotopy.

It is not hard to see that this triangulation and map lift to a triangulation of the oriented Grassmannian $\tilde{G}\left(k, \mathbb{R}^{n}\right)$ and a simplicial map $\tilde{G}\left(k, \mathbb{R}^{n}\right) \rightarrow \widehat{\operatorname{MacP}}(k, n)$.

The hope is that the map $c$ preserves much of the topology of $G\left(k, \mathbb{R}^{n}\right)$. If $k=1$ or 2 , then some element of the homotopy class of $c$ is a homeomorphism. This follows in the case $k=1$ from the Topological Representation Theorem and in the case $k=2$ from Proposition 3.1 below. Because there is a natural duality $\operatorname{MacP}(k, n) \cong \operatorname{MacP}(n-k, n)$ (Section 3.4 in [3]), similar results for $\operatorname{MacP}(n-1, n)$ and $\operatorname{MacP}(n-2, n)$ follow immediately. For general $k$ and $n$, the only previous result on homotopy groups was the observation (see [8]) that $\pi_{0}(\Delta \operatorname{MacP}(k, n))=\pi_{0}(\Delta \widetilde{\operatorname{MacP}}(k, n))=0$. There is ample reason to doubt that $c$ is a homotopy equivalence in general. For instance, $c$ is not surjective (it misses all the nonrealizable oriented matroids), and Mnëv proved that the strata $U_{M}$ are not contractible - in fact, $U_{M}$ can have the topology of any semialgebraic set [7]. (For further bad news on $c$, see [8] and Section 2.4 of [3].)

However, we will be able to show here:

Theorem 1.2. The map $c_{*}: \pi_{i}\left(G\left(k, \mathbb{R}^{n}\right)\right) \rightarrow \pi_{i}(\Delta \operatorname{MacP}(k, n))$ is an isomorphism for $i=1$ and a surjection for $i=2$. 
Corollary 1.1. $\Delta \widetilde{\operatorname{MacP}}(k, n)$ is simply connected. It is the universal double cover of $\Delta \operatorname{MacP}(k, n)$.

There is an obvious embedding

$$
\eta: \operatorname{MacP}(k, n-1) \rightarrow \operatorname{MacP}(k, n)
$$

obtained by adding $n$ as a loop to each oriented matroid of $\operatorname{MacP}(k, n-1)$. Thus we can take the direct limit and define $\operatorname{MacP}(k, \infty)$. This is the classifying space for rank $k$ "matroid bundles," defined in [6]. Theorem 4.3 will imply that $\eta$ gives isomorphisms of homotopy groups for sufficiently large $n$, leading to:

Corollary 1.2. $\eta_{*}: \pi_{i}(\Delta \operatorname{MacP}(k, n-1)) \rightarrow \pi_{i}(\Delta \operatorname{MacP}(k, n))$ is an isomorphism if $n>k(i+2)$ and a surjection for $n>k(i+1)$.

Thus the homotopy groups of $\operatorname{MacP}(k, \infty)$ can be calculated from those for finite MacPhersonians. In terms of nonrealizability, this result suggests that much of the nonrealizability phenomena of general MacPhersonians can be completely understood by studying those with relatively small $n$.

\section{Constructions}

Notation. Let $[n]$ denote the set of integers from 1 to $n$. If $A$ and $B$ are sets, then $A \backslash B$ will denote the set of elements of $A$ not contained in $B$. If $M$ is in $\operatorname{MacP}(k, n)$, then $E(M)$ will denote the set of nonzero elements of $M$, i.e., the elements of $[n]$ on which not all sign vectors are 0 . If $y \in[n]$, then $M$ contains $y$ if $y$ is nonzero in $M$. A basis for $M$ is an independent set of $k$ elements of $M . S^{i}$ will denote the $i$-dimensional sphere and $D^{i}$ will denote the $i$-dimensional ball. If $\sigma$ is a simplex in a simplicial complex $X$, then $\operatorname{star}(\sigma)$ will denote the open star of $\sigma$, that is, $\{\tau \in X: \sigma \subseteq \tau\}$.

If $F$ is a subset of $[n]$, then $M(F)$ will denote the oriented matroid obtained from $M$ by setting all elements of $[n] \backslash F$ equal to 0 . This is a slightly nonstandard notation, since $M(F)$ usually denotes the oriented matroid obtained from $M$ by deleting all elements of $[n] \backslash F$ from the set of elements of $M$. Of course, the two meanings amount to much the same thing, but ours is more convenient in the context of MacPhersonians because if $M \in \operatorname{MacP}(k, n)$ and $F$ is a rank $k$ set of elements in $M$, then under our meaning $M(F)$ will still be in $\operatorname{MacP}(k, n)$.

By the Simplicial Approximation Theorem, any continuous map $S^{i} \rightarrow \Delta \operatorname{MacP}(k, n)$ is homotopic to a simplicial map. It will be convenient here to work with poset maps rather than simplicial maps. If $r: X \rightarrow \Delta \operatorname{MacP}(k, n)$ is a simplicial map, then we get a poset map $s(r)$ from the poset of simplices of $X$ to the poset $\operatorname{MacP}(k, n)$ by defining $s(r)(\sigma)=\max _{v \in \sigma} r(v)$. Conversely, any poset map $s: X \rightarrow \operatorname{MacP}(k, n)$ defines a simplicial map $t(s): \Delta X \rightarrow \Delta \operatorname{MacP}(k, n)$, and $t(s(r))$ is homotopic to $r$. Two poset maps will be said to be homotopic if their corresponding simplicial maps are homotopic. 
Let $X$ be a simplicial complex, let $k$ and $n$ be integers with $1 \leq k \leq n$, and let $s: X \rightarrow \operatorname{MacP}(k, n)$ be a poset map. This section describes several ways to obtain maps $s^{\prime}: X^{\prime} \rightarrow \operatorname{MacP}(k, n)$ homotopic to $s$. In each case $X^{\prime}$ is PL equivalent to $X$.

Definition 2.1. A lowering homotopy of $s$ is a poset map $s^{\prime}: X \rightarrow \operatorname{MacP}(k, n)$ such that $s^{\prime}(\sigma) \leq s(\sigma)$ for each $\sigma \in X$. A raising homotopy of $s$ is a poset map $s^{\prime}: X \rightarrow$ $\operatorname{MacP}(k, n)$ such that $s^{\prime}(\sigma) \geq s(\sigma)$ for each $\sigma \in X$.

In either case, the maps $s^{\prime}$ and $s$ are homotopic by Quillen's Order Homotopy Theorem (see [10] and [2]).

A uniform oriented matroid is an element of $\operatorname{MacP}(k, n)$ in which all $k$-tuples of elements are independent. As one example of a raising homotopy, recall (see [3, Corollary 7.7.9]) that any $M \in \operatorname{MacP}(k, n)$ is the weak map image of a uniform oriented matroid. If $s$ is as above and $\sigma$ is a maximal simplex of $X$ with $s(\sigma)=M$, then we can raise $s$ by sending $\sigma$ to some uniform oriented matroid greater than $M$ and leaving $s(\tau)$ unchanged for all $\tau \neq \sigma$. Thus $s$ is homotopic to a map sending every maximal simplex to a uniform oriented matroid. In fact, the same argument as in [3, Corollary 7.9] shows that for any subset $T$ of the zero set of $M$, we can find $M^{\prime} \geq M$ in $\operatorname{MacP}(k, n)$ with zero set $T$ which is uniform on its nonzero elements. We will use raisings to such $M^{\prime}$ in the proof of Lemma 4.4 .

A trivial oriented matroid is an element of $\operatorname{MacP}(k, n)$ with only $k$ nonzero elements. Every $k$-subset of $[n]$ determines a unique trivial oriented matroid of rank $k$, and thus we will not bother to distinguish the set of elements and the resulting oriented matroid. If $M \in \operatorname{MacP}(k, n)$ and $B$ is a basis of $M$, then $M \geq B$ in $\operatorname{MacP}(k, n)$.

Definition 2.2. Let $X$ be a simplicial complex. A poset map $s: X \rightarrow \operatorname{MacP}(k, n)$ is in canonical form if:

- $s(v)$ is a trivial oriented matroid for each vertex $v$ of $X$; and

- if $\sigma$ is a simplex of $X$, then $E(s(\sigma))=\bigcup_{v \in \sigma} E(s(v))$.

Lemma 2.1. Any poset map $s: X \rightarrow \Delta \operatorname{MacP}(k, n)$ is homotopic to a map in canonical form.

Proof. For each vertex $v$ of $X$, choose a basis $B_{v}$ for $s(v)$. Define a map

$$
\begin{aligned}
s^{\prime}: \quad X & \rightarrow \operatorname{MacP}(k, n), \\
\sigma & \rightarrow s(\sigma)\left(\bigcup_{v \in \sigma} B_{v}\right) .
\end{aligned}
$$

Since deletion induces weak maps, this is a lowering homotopy of $s$.

Let $\sigma$ be a simplex of $X$, and let $B$ be a rank $k$ weak map image of $s(\sigma)$. Consider the simplicial complex $H$ obtained from $X$ by coning $\operatorname{star}(\sigma)$ with a new vertex $v$. Thus $H$ is obtained from $X$ by adding a 0 -simplex $\{v\}$ and simplices $\omega \cup\{v\}$ for all $\omega$ in the 
closed star of $v$. Let $S$ be the map

$$
\begin{aligned}
& S: H \rightarrow \operatorname{MacP}(k, n), \\
& \tau \rightarrow \begin{cases}s(\tau) & \text { if } \tau \in X, \\
s(\omega) & \text { if } \tau=\omega \cup\{v\}, \omega \in \operatorname{star}(\sigma), \\
s(\omega \cup \sigma) & \text { if } \tau=\omega \cup\{v\}, \omega \cup \sigma \in \operatorname{star}(\sigma), \\
B & \text { if } \tau=\{v\} .\end{cases}
\end{aligned}
$$

It is easy to check this is a poset map. $H$ gives a homotopy from $X$ to a stellar subdivision $\tilde{X}$ of $X$, and $S$ gives a homotopy from $s: \Delta X \rightarrow \Delta \operatorname{MacP}(k, n)$ to a map $s^{\prime}: \Delta \tilde{X} \rightarrow$ $\Delta \operatorname{MacP}(k, n)$.

Definition 2.3. The map $s^{\prime}: \Delta \tilde{X} \rightarrow \Delta \operatorname{MacP}(k, n)$ is called a stellar homotopy of $s$ in $\sigma$.

Stellar homotopies will appear in the proofs of Lemmas 3.2, 3.3, and 4.4. In these proofs $B$ will always be a basis of $s(\sigma)$.

Let $\sigma$ and $\tau$ be two abstract simplices, and let $\partial \tau$ denote the simplicial complex of proper faces of $\tau$. The join $\sigma * \partial \tau$ is a PL ball with interior the set of all simplices of the form $\sigma * \hat{\tau}$, with $\hat{\tau} \subset \tau$. The complexes $\sigma * \partial \tau$ and $\partial \sigma * \tau$ both have boundary $\partial \sigma * \partial \tau$. If $X$ is a simplicial complex, a bistellar flip on $X$ consists of replacing a subcomplex $\sigma * \partial \tau$ of $X$ with $\partial \sigma * \tau$. This operation gives a simplicial complex as long as $\sigma$ is not contained in any simplex of $X \backslash(\sigma * \partial \tau)$, and the resulting complex is PL homeomorphic to $X$ as long as $\tau$ is not a simplex of $X$.

Definition 2.4. Let $M$ be an element of $\operatorname{MacP}(k, n)$, and let $\sigma * \partial \tau$ be a subcomplex of $X$ such that $\sigma$ is not contained in any simplex of $X \backslash(\sigma * \partial \tau), \tau$ is not a simplex of $X$, and $s(\omega) \leq M$ for all $\omega$ in $\sigma * \partial \tau$. Let $X^{\prime}$ be the complex obtained from $X$ by replacing $\sigma * \partial \tau$ with $\partial \sigma * \tau$, and define

$$
\begin{aligned}
s^{\prime}: X^{\prime} & \rightarrow \operatorname{MacP}(k, n), \\
\omega & \rightarrow \begin{cases}M & \text { if } \omega=\tau \cup \hat{\sigma}, \text { some } \hat{\sigma} \subset \sigma, \\
s(\omega) & \text { otherwise. }\end{cases}
\end{aligned}
$$

Then $s^{\prime}$ is a bistellar homotopy of $s$.

To see this is a homotopy, let $H$ be the complex obtained from $X$ by adding the simplex $\sigma * \tau$. Let $S$ be the extension of $s$ to $H$ by associating the oriented matroid $M$ to $\sigma * \tau$ and to each face of $\sigma * \tau$ not in $X$. Then $H$ and $S$ give a homotopy between the two maps.

Definition 2.5. Let $M$ be an element of $\operatorname{MacP}(k, n)$ and let $Y$ be a subcomplex of $X$ such that:

- $Y$ is a PL ball; 
- no simplex in the interior of $Y$ is contained in any simplex of $X \backslash Y$; and

- $s(\sigma) \leq M$ for every $\sigma \in Y$.

Let $L$ be the boundary of $Y$, and let $X^{\prime}$ be the complex obtained from $X$ by replacing $Y$ with the cone $L * v$ on a new vertex $v$. Then the map

$$
\begin{aligned}
s^{\prime}: X^{\prime} & \rightarrow \operatorname{MacP}(k, n), \\
\omega & \rightarrow \begin{cases}M & \text { if } \omega=\sigma \cup\{v\}, \text { some } \sigma \in L, \\
s(\omega) & \text { otherwise, }\end{cases}
\end{aligned}
$$

is a coning homotopy of $s$.

This is a homotopy by the same argument as for stellar homotopies.

\section{3. $\pi_{1}(\operatorname{MacP}(k, n))$ and $\pi_{2}(\operatorname{MacP}(k, n))$}

For any $j \geq k$, let $\operatorname{MacP}^{\langle j\rangle}(k, n)$ be the subposet of $\operatorname{MacP}(k, n)$ consisting of oriented matroids with at most $j$ nonzero elements.

The basis exchange axiom for matroids (see [9]) states that if $B_{1}$ and $B_{2}$ are two bases for a matroid $M$ and $a \in B_{1} \backslash B_{2}$, then there is some element $b$ in $B_{2}$ such that $\left(B_{1} \cup\{b\}\right) \backslash\{a\}$ is a basis for $M$. This axiom leads to the following two lemmas on maps of one- and two-dimensional complexes mapping into $\operatorname{MacP}(k, n)$. Unfortunately, the proofs do not appear to extend to higher dimensions. (Whether the results are true in higher dimensions is an open question.)

Lemma 3.2. If $X$ is a one-dimensional simplicial complex and $s: X \rightarrow \operatorname{MacP}(k, n)$ is a poset map, then $s$ is homotopic to a map $s^{\prime}: X^{\prime} \rightarrow \operatorname{Mac}^{\langle k+1\rangle}(k, n)$ via a sequence of stellar homotopies.

Proof. By Lemma 2.1, we may assume $s$ is in canonical form. If $\{v, w\}$ is a 1-simplex of $X$, note that $B_{v}=E(s(v))$ and $B_{w}=E(s(w))$ are both bases of $s(\{v, w\})$. If $s(\{v, w\}) \notin \operatorname{Mac}^{\langle k+1\rangle}(k, n)$, then there is some element $a$ of $B_{v} \backslash B_{w}$, and by the basis exchange axiom there is some $b \in B_{w}$ so that $B=\left(B_{v} \cup\{b\}\right) \backslash\{a\}$ is a basis for $g(\{v, w\})$. Thus by taking a stellar homotopy in $\{v, w\}$ using the basis $B$, we subdivide this 1-simplex into two 1-simplices whose associated oriented matroids have strictly fewer nonzero elements than $s(\{v, w\})$. By taking further such stellar homotopies, we can subdivide until all 1-simplices in the subdivision are mapped to $\operatorname{MacP}^{\langle k+1\rangle}(k, n)$.

Lemma 3.3. If $X$ is a two-dimensional simplicial complex and $s: X \rightarrow \operatorname{MacP}(k, n)$ is a poset map, then $s$ is homotopic to a map $s^{\prime}: X^{\prime} \rightarrow \operatorname{MacP}^{\langle k+2\rangle}(k, n)$.

Proof. Every minimal element of $\operatorname{MacP}(k, n)$ is in $\operatorname{MacP}^{\langle k+2\rangle}(k, n)$. For any $\sigma \in X$, define $\rho(\sigma)$ to be 0 if $s(\sigma) \in \operatorname{MacP}^{\langle k+2\rangle}(k, n)$, and the maximal length of a chain in $\operatorname{MacP}(k, n)$ from $s(\sigma)$ to a maximal element of $\operatorname{MacP}^{\langle k+2\rangle}(k, n)$ otherwise. The proof is by induction on $\max _{\sigma \in X} \rho(\sigma)$ : if this maximum is greater than 0 , we give a homotopy of $s$ to reduce it. 
Again, assume $s$ is in canonical form. By the previous lemma, there exists a sequence of stellar homotopies on the 1-skeleton $X^{(1)}$ of $X$ resulting in a map $X^{(1)} \rightarrow$ $\operatorname{MacP}^{\langle k+1\rangle}(k, n)$. We can carry out the same sequence of stellar homotopies in $X$, resulting in a subdivision $X_{1}$ of $X$ and $s_{1}: X_{1} \rightarrow \operatorname{MacP}(k, n)$ such that, for every 2-simplex $\sigma$ of $X$, the subdivision of the boundary of $\sigma$ maps to $\operatorname{MacP}^{\langle k+1\rangle}(k, n)$. Then we may take a lowering homotopy to put the map into canonical form.

Let $\sigma$ be a simplex of $X$ with $\rho(\sigma)$ maximal. If the subdivision $L$ of the boundary of $\sigma$ has only three 1-simplices, then $|E(s(\sigma))|=\left|\bigcup_{\tau \in \sigma} E\left(s_{1}(\tau)\right)\right| \leq k+2$. In fact, even if exactly one of these three 1-simplices did not map to $\operatorname{MacP}^{\langle k+1\rangle}(k, n)$, we would still have $|E(s(\sigma))|=k+2$. Also, if for every such $\sigma$ and every $\tau$ in the subdivision of $\sigma$ we have $\rho(\tau)<\rho(\sigma)$, then by the induction hypothesis $s_{1}$ is homotopic to a map to $\operatorname{MacP}^{\langle k+2\rangle}(k, n)$.

Otherwise, the vertices of $L$ have a cyclic order $v_{1}, v_{2}, \ldots, v_{m}$, with $m>3$. First, take a coning homotopy replacing the subdivision of $\sigma$ with a cone $L * u$, associating the oriented matroid $s(\sigma)$ to each simplex in the interior of $L * u$.

The next step is to give a sequence of stellar, bistellar, and raising homotopies in $\operatorname{star}_{L * u}(u)$ resulting in a subdivision $S$ of $L * u$ and map $s_{2}: S \rightarrow \operatorname{MacP}(k, n)$ so that:

- $\left(\operatorname{link}_{S}(u)\right)^{0}=\left\{v_{1}^{\prime}, v_{2}, v_{3}, \ldots, v_{m-1}\right\}$, where $v_{1}^{\prime}$ is a vertex taking the place of $v_{1}$ in the cyclic order;

- $s_{2}(\tau)<s(\sigma)$ for every simplex $\tau$ in $S \backslash \operatorname{star}_{S}(u)$; and

- all 1-simplices of $\operatorname{link}_{S}(u)$ except possibly $\left\{v_{1}^{\prime}, v_{2}\right\}$ map to $\operatorname{MacP}^{\langle k+1\rangle}(k, n)$.

This sequence begins with a bistellar homotopy removing $v_{m}$ from link $(u)$. The subcomplex $\operatorname{star}\left(\left\{u, v_{m}\right\}\right)$ can be written $\left\{u, v_{m}\right\} * \partial\left\{v_{1}, v_{m-1}\right\}$. Because $L * u$ is a 2-ball and each oriented matroid associated to $L * u$ is less than or equal to $s(\sigma)$, there is a bistellar homotopy replacing $\left\{u, v_{m}\right\} * \partial\left\{v_{1}, v_{m-1}\right\}$ with $\partial\left\{u, v_{m}\right\} *\left\{v_{1}, v_{m-1}\right\}$. (This is the first operation pictured in Fig. 1.) We then take a lowering homotopy to put the resulting map in canonical form.

Since $\left|E\left(s_{2}\left(\left\{v_{1}, v_{m}\right\}\right)\right)\right| \leq k+1$ and $\left|E\left(s_{2}\left(\left\{v_{m-1}, v_{m}\right\}\right)\right)\right| \leq k+1$, the oriented matroid associated to the simplex $\left\{v_{m-1}, v_{m}, v_{1}\right\}$ will be in $\operatorname{MacP}^{\langle k+2\rangle}(k, n)$. If the oriented matroid associated to $\left\{v_{m-1}, v_{1}\right\}$ has only $k+1$ nonzero elements, then we have $S$. Otherwise, we take a further stellar homotopy to get $S$, as follows.

The stellar subdivision will be by a vertex $v_{1}^{\prime}$ in the simplex $\left\{u, v_{1}\right\}$. (This is the second operation pictured in Fig. 1.) To decide what oriented matroid to associate to $v_{1}^{\prime}$, there are three cases to consider. (The cases are necessary to ensure that the two simplices

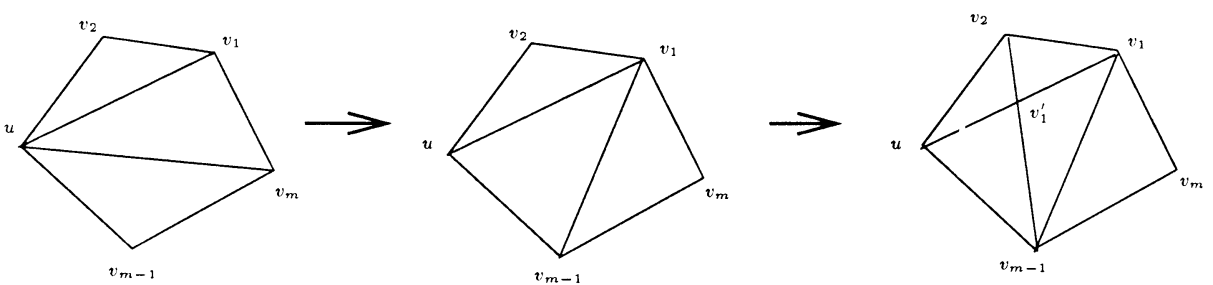

Fig. 1. Steps in the proof of Lemma 4.4. 
$\left\{v_{1}, v_{1}^{\prime}, v_{2}\right\}$ and $\left\{v_{1}, v_{1}^{\prime}, v_{m-1}\right\}$ end up with oriented matroids strictly less than $s(\sigma)$.) Let $a, b$ be the two elements of $s_{2}\left(v_{m-1}\right) \backslash s_{2}\left(v_{1}\right)$.

- If $a \notin s_{2}\left(v_{2}\right)$, then by the basis exchange axiom there is an element $c$ of $s_{2}\left(v_{1}\right) \backslash s_{2}\left(v_{m-1}\right)$ such that $B=s(\sigma)\left(\left(E\left(s_{2}\left(v_{1}\right)\right) \cup\{b\}\right) \backslash\{c\}\right)$ is a basis for $s_{2}\left(\left\{u, v_{1}\right\}\right)$. Associate the oriented matroid $B$ to $v_{1}^{\prime}$. Then the oriented matroid associated to $\left\{v_{1}, v_{1}^{\prime}, v_{m-1}\right\}$ is in $\operatorname{MacP}^{\langle k+2\rangle}(k, n)$, and the oriented matroid associated to $\left\{v_{1}, v_{1}^{\prime}, v_{2}\right\}$ is 0 on $a$. Hence both are strictly less than $s(\sigma)$.

- Similarly, if $b \notin s_{2}\left(v_{2}\right)$, then we associate to $v_{1}^{\prime}$ a basis not containing $b$ in the same way.

- If both $a$ and $b$ are in $s_{2}\left(v_{2}\right)$, then we begin by taking the same stellar homotopy as in the first case. The simplex $\left\{v_{1}, v_{1}^{\prime}, v_{m-1}\right\}$ is then associated to an element of $\operatorname{Mac}^{\langle k+2\rangle}(k, n)$ just as before. Recall that $s_{2}\left(\left\{v_{1}, v_{2}\right\}\right)$ is strictly less than $s(\sigma)$. Also note that for each face of the closed simplex $\left\{v_{1}, v_{1}^{\prime}, v_{2}\right\}$ which is not a face of $\left\{v_{1}, v_{2}\right\}$, the associated oriented matroid is obtained from $s(\sigma)$ by sending to zero some subset of $E\left(s_{2}\left(\left\{v_{1}, v_{2}\right\}\right)\right)$. It is easy to check that $s(\sigma) \geq s_{2}\left(\left\{v_{1}, v_{2}\right\}\right)$ implies $s(\sigma)(A) \geq s_{2}\left(\left\{v_{1}, v_{2}\right\}\right)(A)$ for any subset $A$ of $E(\sigma)$. W can thus do a lowering homotopy on the closed simplex $\left\{v_{1}, v_{1}^{\prime}, v_{2}\right\}$ by associating to each simplex the appropriate deletion of $s_{2}\left\{v_{1}, v_{2}\right\}$, giving our final $S$.

Note that no step in this process used the fact that $s_{2}\left(\left\{v_{1}, v_{2}\right\}\right)$ was in $\operatorname{MacP}^{\langle k+1\rangle}(k, n)$ it was only required that $s_{2}\left(\left\{v_{1}, v_{2}\right\}\right)<s(\sigma)$. Also note that in the resulting $S$ we have $s_{2}\left(\left\{v_{1}^{\prime}, v_{2}\right\}\right)<s(\sigma)$. Thus by continuing in the same way we can remove $v_{m-1}, v_{m-2}, \ldots, v_{4}$ from the link of $u$, resulting in a complex $S^{\prime}$ with $\operatorname{link}_{S^{\prime}}(u)$ having only three 1-simplices, with two of these mapping to $\operatorname{MacP}^{\langle k+1\rangle}(k, n)$ (and hence $\operatorname{star}(u)$ maps to $\operatorname{MacP}^{\langle k+2\rangle}(k, n)$, as before), and with all simplices $S^{\prime}$ mapping to oriented matroids less than $s(\sigma)$. Also note that none of these homotopies within the subdivision of $\sigma$ affect any simplices outside of the subdivision of $\sigma$. Thus we can carry out this process on the subdivision of each 2-simplex $\sigma$ with $\rho(\sigma)$ maximal, resulting in a map $s^{\prime}$ from a subdivision of $X$ to $\operatorname{MacP}(k, n)$ giving a smaller maximal value of $\rho$. Thus by induction we can homotope $s$ to $\tilde{s}: \tilde{X} \rightarrow \operatorname{MacP}^{\langle k+2\rangle}(k, n)$.

The remaining ingredient we need to prove Theorem 1.2 is an assurance that the preimage of $\operatorname{MacP}^{\langle k+2\rangle}(k, n)$ in the Grassmannian is well behaved. Let $G^{\langle i\rangle}(k, n)=$ $\bigcup_{M \in \operatorname{MacP}^{(i)}(k, n)} U_{M}$. This is the set of all $k$-planes in $\mathbb{R}^{n}$ contained in the intersection of $n-i$ coordinate hyperplanes.

Proposition 3.1. $\quad c: G^{\langle k+2\rangle}(k, n) \rightarrow \Delta \operatorname{MacP}^{\langle k+2\rangle}(k, n)$ is a homotopy equivalence.

(In fact, we will give an element of the homotopy class $c$ which is a homeomorphism.)

Proof. We will show that for any $M \in \operatorname{MacP}^{\langle k+2\rangle}(k, n)$ :

1. the closure $\bar{U}_{M}$ equals $\bigcup_{M^{\prime} \in(\operatorname{MacP}(k, n)) \leq M} U_{M^{\prime}}$;

2. $U_{M}$ is contractible; and

3. $\bar{U}_{M}$ is a topological ball. 
(Note: Each of these statements is false without the condition $M \in \operatorname{MacP}^{\langle k+2\rangle}(k, n)$ (see Section 2.4 in [3]).)

Thus the stratification $\left\{U_{M}: M \in \operatorname{MacP}^{\langle k+2\rangle}(k, n)\right\}$ is a regular cell complex, and so its barycentric subdivision is a simplicial complex homeomorphic to $\operatorname{MacP}^{\langle k+2\rangle}(k, n)$.

Assume without loss of generality that $E(M) \subseteq[k+2]$. Then $U_{M^{\prime}} \subseteq G\left(k, \mathbb{R}^{k+2}\right)$ for each $M^{\prime} \leq M$. Under the duality homeomorphism $G\left(k, \mathbb{R}^{k+2}\right) \rightarrow G\left(2, \mathbb{R}^{k+2}\right)$, the stratum $U_{M^{\prime}}$ is taken to the stratum $U_{\left(M^{\prime}\right)^{*}}$ of the dual oriented matroid $\left(M^{\prime}\right)^{*} \in$ $\operatorname{MacP}(2, k+2)$. Thus it suffices to prove the above statements for $M \in \operatorname{MacP}(2, n)$ for all $n$.

Assume without loss of generality that the first two elements of $M$ are nonzero. Any $V \in U_{M}$ can be given uniquely as the row space of a matrix

$$
m=\left(\begin{array}{ccccc}
1 & 0 & v_{13} & \cdots & v_{1 n} \\
0 & 1 & v_{23} & \cdots & v_{2 n}
\end{array}\right)
$$

The lines perpendicular to the columns $(1,0),(0,1), \mathbf{v}_{3}, \ldots, \mathbf{v}_{n}$ of $m$ are an arrangement of oriented lines through the origin in $\mathbb{R}^{2}$ with oriented matroid $M$. Thus $U_{M} \cong$ $\left\{\left(\mathbf{v}_{3}, \ldots, \mathbf{v}_{n}\right):\left\{(1,0)^{\perp},(0,1)^{\perp}, \mathbf{v}_{3}^{\perp}, \ldots, \mathbf{v}_{n}^{\perp}\right\}\right.$ represents $\left.M\right\}$.

$M$ is completely determined by the cyclic order of the nonzero elements of $\left\{(1,0),(-1,0),(0,1),(0,-1), \mathbf{v}_{3},-\mathbf{v}_{3}, \ldots, \mathbf{v}_{n},-\mathbf{v}_{n}\right\}$. Thus $U_{M}$ can be seen as a product of open intervals, hence is contractible. $V^{\prime} \in U_{M^{\prime}}$ is in the closure of $U_{M}$ if the cyclic order for $M^{\prime}$ is obtained from the order for $M$ by making some neighboring elements parallel and setting some elements to 0 . For rank 2 oriented matroids, this is equivalent to $M^{\prime}$ being a specialization of $M$. Thus $\bar{U}_{M}=\bigcup_{M^{\prime} \in(\operatorname{MacP}(k, n))_{<M}} U_{M^{\prime}}$, and $\bar{U}_{M}$ is homeomorphic to a product of closed intervals, hence is a topological ball.

Together, these statements give the proof of Theorem 1.2, as follows.

Proof. By Lemmas 3.2 and 3.3, any poset map $S^{i} \rightarrow \operatorname{MacP}(k, n)$, with $i=1$ or 2, is homotopic to a map $S^{i} \rightarrow \operatorname{MacP}^{\langle k+2\rangle}(k, n)$. Thus, by Proposition 3.1, any such map can be written as a composition $S^{i} \rightarrow G\left(k, \mathbb{R}^{n}\right) \stackrel{c}{\rightarrow} \operatorname{MacP}(k, n)$, and so $c$ gives a surjection in $\pi_{1}$ and $\pi_{2}$. To see injectivity in $\pi_{1}$, consider a map $f: S^{1} \rightarrow G\left(k, \mathbb{R}^{n}\right)$ such that $c \circ f: S^{1} \rightarrow \operatorname{MacP}(k, n)$ is homotopically trivial. Then $f$ is homotopic to a map to the 1skeleton of any Schubert cell decomposition of $G\left(k, \mathbb{R}^{n}\right)$. If we take such a decomposition corresponding to a flag given by coordinate hyperplanes, then the 1-skeleton will map under $c$ to $\operatorname{MacP}^{\langle k+1\rangle}(k, n)$. Thus we may assume that $c \circ f\left(S^{1}\right) \subseteq \operatorname{MacP}^{\langle k+1\rangle}(k, n)$. Since $c \circ f$ is homotopically trivial, there exists a map $h: D^{2} \rightarrow \operatorname{MacP}(k, n)$ from the 2-ball to $\operatorname{MacP}(k, n)$ restricting to $c \circ f$ on $S^{1}$. The proof of Lemma 3.3 gives a homotopy of $h$ to a map $h^{\prime}: D^{2} \rightarrow \operatorname{MacP}^{\langle k+2\rangle}(k, n)$ such that $\left.h\right|_{S^{1}}=\left.h^{\prime}\right|_{S^{1}}$. Since $h^{\prime}$ can be written as a composition $D^{2} \rightarrow G\left(k, \mathbb{R}^{n}\right) \stackrel{c}{\rightarrow} \operatorname{MacP}(k, n)$, the map $f$ is homotopically trivial.

Given that $c$ gives a surjection in $\pi_{1}$, we could also see that this $c_{*}$ is an isomorphism by noting that $\pi_{1}\left(G\left(k, \mathbb{R}^{n}\right)\right)=\mathbb{Z}_{2}$ and that $\Delta \operatorname{MacP}(k, n)$ has a connected double cover $\Delta \widetilde{\operatorname{MacP}}(k, n)$, and hence $\left|\pi_{1}(\Delta \operatorname{MacP}(k, n))\right| \geq 2$. We can now conclude that $\Delta \widetilde{\operatorname{MacP}}(k, n)$ is the universal cover of $\Delta \operatorname{MacP}(k, n)$, hence is simply connected. 


\section{Stability of $\pi_{i}(\operatorname{MacP}(k, n))$}

Corollary 1.2 will follow from the following theorem:

Theorem 4.3. Let $s: X \rightarrow \operatorname{MacP}(k, n)$ be a poset map from an i-dimensional simplicial complex to the MacPhersonian, where $k>1$ and $i>0$. Then $s$ is homotopic to a map $s^{\prime}: X^{\prime} \rightarrow \operatorname{MacP}(k, k(i+1))$.

Lemma 4.4. Let $s: X \rightarrow \operatorname{MacP}(k, n)$ be a poset map, where $\operatorname{dim}(X)=i>0$ and $k>1$. Let $m$ be an element of $[i]$. Let $\sigma$ be a codimension $m$ simplex of $X$ and let $e$ be the largest element of $\bigcup_{\tau \in \operatorname{link}(\sigma)} E(s(\tau))$. If e $>(k-1) i+m$, then there is a sequence of stellar homotopies in $\operatorname{star}(\sigma)$ such that:

1. $E(s(x)) \subseteq[e-1]$ for every vertex $x$ introduced; and

2. the resulting complex $X^{\prime}$ has $e \notin \bigcup_{\tau \in \operatorname{link}_{X^{\prime}}(\sigma)} E(s(\tau))$.

Proof. By induction on $m$ : if $m=1$, then $k>1$ and $i>0$ implies $(k-1) i+m \geq k$. Let $\sigma \cup\{v\}$ be a simplex of $\operatorname{star}(\sigma)$ with $e \in M(v)$. Since $\sigma \cup\{v\}$ is a maximal simplex, we may assume $s(\sigma \cup\{v\})$ is uniform on its nonzero elements and $[k] \subseteq E(s(\sigma \cup\{v\}))$. Thus $[k]$ is a basis for $s(\sigma \cup\{v\})$. We take a stellar homotopy by adding a vertex in the interior of $\sigma \cup\{v\}$ and assigning the oriented matroid $[k]$ at that vertex. By taking such a stellar homotopy for each such $\sigma \cup\{v\}$, we can get $e$ out of $\operatorname{link}(\sigma)$.

Now, assuming we can find such a sequence for codimension $m-1$ simplices, consider a codimension $m$ simplex $\sigma$. If $v$ is a vertex in $\operatorname{link}(\sigma)$ with $e \in E(s(v))$, then the goal is to find a sequence of stellar subdivisions in $\operatorname{star}(\sigma)$ to get $v$ out of $\operatorname{link}(\sigma)$.

If $s(\sigma \cup\{v\})$ has a basis $B$ not containing $e$, then taking a stellar subdivision of $\sigma \cup\{v\}$ and associating $B$ to this new vertex achieves this goal.

If not, then we begin by using our induction hypothesis in a weak way. Take a sequence of stellar homotopies in $\operatorname{star}(\sigma \cup\{v\})$ resulting in $X^{\prime}$ with $\bigcup_{\tau \in \operatorname{link}_{X^{\prime}}(\sigma \cup\{v\})} E(s(\tau)) \subseteq$ $[(k-1) i+m]$. The induction hypothesis allows us to remove one more element from this union-we will choose that element later.

Since every basis for $s(\sigma \cup\{v\})$ contains $e$, we know that $e$ must be an element of $M(w)$ for every vertex $w$ of $\sigma \cup\{v\}$. Since $|\sigma| \leq i-1$, we know that $\left|\bigcup_{w \in \sigma \cup\{v\}} E(w)\right| \leq$ $(k-1)(1+|\sigma|)+1 \leq(k-1) i+1<(k-1) i+m$. So let $f$ be an element of $[(k-1) i+m] \backslash E(s(\sigma))$. The induction hypothesis gives stellar homotopies in $\operatorname{star}(\sigma \cup\{v\})$ leading to a complex $X^{\prime \prime}$ with $\bigcup_{\tau \in \operatorname{link}_{X^{\prime \prime}}(\sigma \cup\{v\})} E(\tau) \subseteq[(k-1) i+m] \backslash\{f\}$.

We now extend $s(\tau)$ for each $\tau \in \operatorname{star}_{X^{\prime \prime}}(\sigma \cup\{v\})$ by adding $f$ parallel to $e$. This gives a map $s^{\prime}$ which is homotopic to $s$ by a raising homotopy. Now $s^{\prime}(\sigma \cup\{v\})$ contains a basis $B$ not containing $e$. So by taking a stellar subdivision in $\sigma \cup\{v\}$ and associating $B$ to the new vertex, we get $v$ out of $\operatorname{link}(\sigma)$.

In the process of getting $v$ out of $\operatorname{link}(\sigma)$, we have introduced no new vertices whose oriented matroids have elements greater than or equal to $e$, and so by this process we get $e$ out of $\operatorname{link}(\sigma)$.

We have now done the hard work for proving Theorem 4.3. 
Proof. We may assume $s$ to be in canonical form. Let $e$ be the largest element in $\bigcup_{v \in X} E(s(v))$, and assume $e>k(i+1)$. Let $\sigma$ be a 0 -simplex of $X$ with $e \in E(s(\sigma))$. Then by Lemma 4.4, we may assume $\bigcup_{\tau \in \operatorname{link}(\sigma)} E(s(\tau)) \subseteq[k i]$. Since $|E(s(\sigma))|=k$ and $E(s(\sigma))$ contains an element not in $[k(i+1)]$, there is some $f$ in $[k(i+1)] \backslash$ $\left(\bigcup_{\tau \in \operatorname{star}(\sigma)} E(s(\tau))\right)$. We obtain a map $s^{\prime}$ by adding $f$ parallel to $e$ at the oriented matroid corresponding to each simplex of $\operatorname{star}(\sigma)$. This is a raising homotopy of $s$, and $E\left(s^{\prime}(\sigma)\right)$ has a basis $B$ not containing $e$. Using this $B$, we take a stellar homotopy at $\sigma$. In this way we can remove $e$ from the image of each 0 -simplex of $X$. Any lowering homotopy to canonical form will send each simplex of $\operatorname{star}(\sigma)$ to an oriented matroid which is 0 on $e$.

The proof of Corollary 1.2 follows immediately:

Proof. If $k=1$, then $G\left(k, \mathbb{R}^{n}\right)$ is homeomorphic to $\Delta \operatorname{MacP}(k, n)$, as stated earlier, and if $i=0$, then $\pi_{i}(\Delta \operatorname{MacP}(k, n))=0$ for all $k$ and $n$. Otherwise, if $n>k(i+1)$, then Theorem 4.3 says that any map $S^{i} \rightarrow \operatorname{MacP}(k, n)$ is homotopic to a map to the image of $\eta: \operatorname{MacP}(k, n-1) \rightarrow \operatorname{MacP}(k, n)$, and so $\eta_{*}: \pi_{i}(\operatorname{MacP}(k, n-1)) \rightarrow \pi_{i}(\operatorname{MacP}(k, n))$ is surjective. If $n>k(i+2)$, let $f: S^{i} \rightarrow \operatorname{MacP}(k, n-1)$ be a poset map such that $\eta \circ f$ is homotopically trivial. By the above remarks, we may assume $f\left(S^{i}\right) \subseteq \operatorname{MacP}(k, k(i+1))$. Then there exists a map $h: D^{i+1} \rightarrow \operatorname{MacP}(k, n)$ such that $\left.h\right|_{S^{i}}=\eta \circ f$. The proof of Theorem 4.3 gives a homotopy from $h$ to a map $h^{\prime}: D^{i+1} \rightarrow \operatorname{MacP}(k, k(i+2)) \subseteq$ $\operatorname{MacP}(k, n-1)$ such that $\left.h^{\prime}\right|_{S^{i}}=\left.h\right|_{S^{i}}$. Thus $f$ is homotopically trivial.

\section{Acknowledgments}

Many thanks to Eric Babson, James Davis, and Robert MacPherson for their suggestions.

\section{References}

1. E. Babson, A combinatorial flag space, Ph.D. thesis, MIT, 1993.

2. A. Björner, Topological methods, In: Handbook of Combinatorics (R. Graham, M. Grötschel, and L. Lovász, eds.), North-Holland, Amsterdam, 1995.

3. A. Björner, M. Las Vergnas, B. Sturmfels, N. White, and G. Ziegler, Oriented Matroids, Encyclopedia of Mathematics and its Applications, Cambridge University Press, Cambridge, 1992.

4. J. Folkman and J. Lawrence, Oriented matroids, J. Combin. Theory, Ser. B, 25 (1978), 283-289.

5. M. Las Vergnas, Bases in oriented matroids, J. Combin. Theory, Ser. B, 25 (1978), 283-289.

6. R. MacPherson, Combinatorial differential manifolds, Topological Methods in Modern Mathematics: A Symposium in Honor of John Milnor's Sixtieth Birthday, Publish or Perish, New York, 1993.

7. N. Mnëv, The universality theorems on the classification problem of configuration varieties and convex polytope varieties, Topology and Geometry-Rohlin Seminar (O. Ya Viro, ed.), Lecture Notes in Mathematics, vol. 1346, Springer-Verlag, New York, 1988, pp. 527-544.

8. N. Mnëv and G. Ziegler, Combinatorial models for the finite-dimensional Grassmannians, Discrete and Comput. Geom., 10 (1993), 241-250.

9. J. Oxley, Matroid Theory, Oxford University Press, Oxford, 1992.

10. D. Quillen, Homotopy properties of the poset of non-trivial p-subgroups of a group, Adv. in Math., 28, 101-128.

Received November 4, 1996, and in revised form April 22, 1997. 